\title{
Formation Principles and Ligand Dynamics of Nanoassemblies of CdSe Quantum Dots and Functionalised Dye Molecules
}

Thomas Blaudeck, ${ }^{[a, b]}$ Eduard I. Zenkevich, ${ }^{[a, c]}$ Mohamed Abdel-Mottaleb, ${ }^{[a, d, e]}$

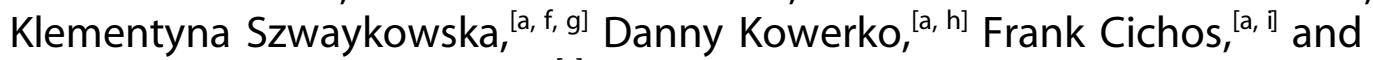

Christian von Borczyskowski*[a]

Functional dye molecules, such as porphyrins, attached to CdSe quantum dots (QDs) through anchoring meso-pyridyl substituents, form quasi-stable nanoassemblies. This fact results in photoluminescence (PL) quenching of the QDs both due to Förster resonance energy transfer (FRET) and the formation of non-radiative surface states under conditions of quantum confinement (non-FRET). The formation process is in competition with the ligand dynamics. At least two timescales are found for the formation of the assemblies: 1) one faster than $60 \mathrm{~s}$ attributed to saturation of empty attachment sites and 2) one slower than $600 \mathrm{~s}$, which is attributed to a reorganisation of the tri-n-octylphosphine oxide (TOPO) ligand shell. Finally, this process results in almost complete exchange of the TOPO shell by porphyrin dye molecules. Following a Stern-
Volmer analysis, we established a microscopic description of PL quenching and assembly formation. Based on this formalism we determined the equilibrium constant for assembly formation between QDs and the pyridyl-functionalised dye molecules to be $K \approx 10^{5}-10^{7} \mathrm{M}^{-1}$, which is several orders of mag nitude larger than that of the TOPO ligands. Our results give additional insights into the non-FRET PL quenching processes involved and show that the QD surface is inhomogeneous with respect to the involved attachment and detachment processes. In comparison with other methods, such as NMR spectroscopy, the advantage of our approach is that ligand dynamics can be investigated at extremely low ratios of dye molecules to QDs.

\section{Introduction}

Optical properties of semiconductor quantum dots (QD) have been investigated intensively during the last two decades, ${ }^{[1-4]}$ including the roles of design of core-shell systems, ${ }^{[5]}$ ligand chemistry $^{[6-12]}$ or surface functionalisation. ${ }^{[13-18]}$ Tuneable band [a] Dr. T. Blaudeck, Prof. Dr. E. I. Zenkevich, Prof. Dr. M. Abdel-Mottaleb, K. Szwaykowska, Dr. D. Kowerko, Prof. Dr. F. Cichos,

Prof. Dr. C. von Borczyskowski

Chemnitz University of Technology

Institute of Physics and Center for Nanostructured

Materials and Analytics (nanoMA)

Reichenhainer Str. 70

09107 Chemnitz (Germany)

E-mail:borczyskowski@physik.tu-chemnitz.de

[b] Dr. T. Blaudeck

Linköping University, Department of Science and Technology (ITN)

Organic Electronics

Bredgatan 34

60174 Norrköping (Sweden)

[c] Prof. Dr. E. I. Zenkevich

National Technical University of Belarus

Department of Information Technologies and Robototechnique

65 Nezavisimosti Ave

Minsk 220013 (Belarus)

[d] Prof. Dr. M. Abdel-Mottaleb

Nile University, Center for Nanotechnology Research

Km 28 Cairo-Alexandria Desert Road (Smart Village B2)

11757 Cairo (Egypt)

[e] Prof. Dr. M. Abdel-Mottaleb

Sabry Corporation for Research and Development

4 Al-Sabbagh Road (El Korba)

11757 Cairo (Egypt)

\author{
[f] K. Szwaykowska \\ California Institute of Technology, Department of Physics \\ 103-33 \\ Pasadena, CA 91125 (USA) \\ [g] K. Szwaykowska \\ Georgia Institute of Technology \\ Department of Electrical and Computer Engineering \\ 777 Atlantic Drive NW \\ Atlanta, GA 30332-0250, GA (USA) \\ [h] Dr. D. Kowerko \\ University of Zürich, Institute for Inorganic Chemistry \\ Winterthurerstr. 190 \\ 8057 Zürich (Switzerland) \\ [i] Prof. Dr. F. Cichos \\ University of Leipzig \\ Institute of Experimental Physics I, Molecular Nanophotonics \\ Linnéstr. 5 \\ 04103 Leipzig (Germany) \\ Supporting information for this article is available on the WWW under \\ http://dx.doi.org/10.1002/cphc.201100711.
}


position and high quantum yields of photoluminescence (PL) are of crucial importance for envisaged applications. ${ }^{[19,20]}$ Colloidal QDs in solution are exposed to a number of dynamic processes related to the interface that affect their optical properties. To name a few, this can be the adsorption of spurious molecules ${ }_{;}^{[6,7]}$ attachment and detachment of protective chelating ligands, ${ }^{[13]}$ or molecules chemisorbed ${ }^{[17,18]}$ to the QD surface, or participation of the QDs in hybrid complexes (nanoassemblies) with functionalised molecules such as dyes ${ }^{[14-16,23]}$ or proteins ${ }^{[24,25]}$ molecules. In general, a surface reconstruction from "mobile traps"[26] can be invoked by any of the ligands mentioned. Frenzel et al. calculated that surface states split off from the band edge of the valence and conduction band in the case of non-passivated QDs. ${ }^{[27]}$ Thus, the type and concentration of ligands controls the optical energies of surface states and their influence on the optical properties of QDs, which is important for any application involving sensing capabilities. ${ }^{[17,18]}$ Furthermore, these dynamics are influenced by temperature $^{[28]}$ and the dielectric properties of the solvent. ${ }^{[29]}$ Stimulated by recent results on the dynamics of the exchange of organic ligands, ${ }^{[6-12]}$ we extend the reported concept in such a way that we consider a dynamic equilibrium between the $\mathrm{QD}$, the functionalised dye molecules and tri- $n$-octylphosphine oxide (TOPO) ligands. Knowledge of ligand dynamics and surface functionalisation can play an important role in various technological fields, for example, for the fabrication of nanostructured inks for solution-processed photovoltaics ${ }^{[30]}$ or printed semiconductor layers in flexible electronics. ${ }^{[31]}$

Herein, we demonstrate that dye molecules that quench the $\mathrm{PL}$ of the QD in controlled use are an indirect measure of the ligand dynamics at the QD surface. Our focus lies on heteronanoassemblies from uncapped $\mathrm{CdSe}$, as well as CdSe/ZnS QDs and $(5,10,15,20)$-tetra-meta-pyridylporphyrin $\left[(m-\mathrm{Pyr})_{4}-\right.$ $\left.\mathrm{H}_{2} \mathrm{P}\right)$ or $\left.\mathrm{H}_{2} \mathrm{P}\right]$ dye molecules. Figure $1 \mathrm{~A}$ shows a schematic representation of such a QD- $\mathrm{H}_{2} \mathrm{P}$ hetero-nanoassembly, ${ }_{1}^{[32]}$ consisting of a QD with a TOPO surfactant layer and one $\mathrm{H}_{2} \mathrm{P}$ mole- cule attached through its meso-pyridyl rings almost perpendicularly to the QD surface. ${ }^{[32]}$

Due to the simultaneous observation of FRET from the QD to the dye, which serves as an indicator of the formation of an assembly and hence the status of the surface coverage, we have direct access to surface-related processes. As outlined herein, the chemical topography of the CdSe and CdSe/ZnS QDs with respect to assembly formation is not at all as homogenous as the picture of a QD as a spherical object could explain (Figure 1 B).

PL quenching within such nanoassemblies has been investigated in detail. In general, both FRET ${ }^{[14-16,32-37]}$ and non-FRET quenching mechanisms ${ }^{[32-37]}$ significantly different from photoinduced charge transfer ${ }^{[32,36]}$ were identified. At low molar ratios, $x=[\mathrm{QD}] /\left[\mathrm{H}_{2} \mathrm{P}\right]$ (in which the square brackets denote the concentration of the species), the additional quenching mechanism could be identified as "leaking" of the excitonic wave function beyond the core or even ZnS shell of the CdSe QD, accompanied by surface trap formation induced by the attached dyes. ${ }^{[32,33]}$ However, at that time, we were not able to assign PL quenching processes for higher molar ratios. This task is discussed in the following, assuming that titration experiments can indeed be a means to investigate the chemical topography of the QDs and provide insights into ligand and dye dynamics in relation to $\mathrm{PL}$ quenching processes, which is of crucial importance for photoinduced processes in QD-dye assemblies.

\section{Results}

\subsection{PL Emission and Absorption Spectra}

Since optical spectra related to various titration experiments on $\mathrm{QD}-\mathrm{H}_{2} \mathrm{P}$ assemblies have already been published recently in detail, ${ }^{[32-35]}$ in Figure 2, we exemplify the decrease of the PL emission spectrum of uncapped CdSe QDs together with the increase of the fluorescence of $(m-\mathrm{Pyr})_{4}-\mathrm{H}_{2} \mathrm{P}$ during a titration experiment. The titration results in a PL quenching of the CdSe QDs which can be attributed to the formation of nanoassemblies through the pyridyl groups of the porphyrin. ${ }^{[32]}$ The inset shows the apparent isosbestic point on an enlarged scale. It can be clearly seen that this is not a true isosbestic point, which indicates that PL decrease and fluorescence increase are not uniquely related to each other. There is also a small blueshift for the QD emission upon assembly formation.

An interesting indicator of assembly formation is the change in the porphyrin Soret band 


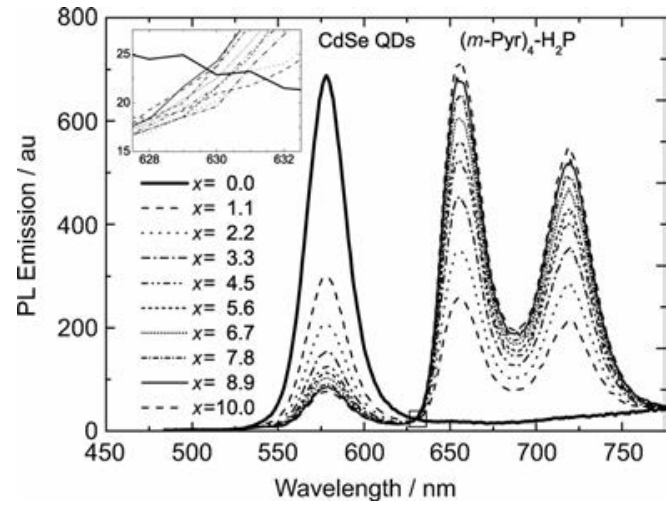

Figure 2. $\mathrm{PL}$ spectra of $\mathrm{CdSe} Q \mathrm{QDs}$ upon titration of $(m-\mathrm{Pyr})_{4}-\mathrm{H}_{2} \mathrm{P}$ molecules in toluene at various molar ratios, $x$. Upon increasing $\mathrm{H}_{2} \mathrm{P}$ concentration, the $\mathrm{PL}$ intensity decreases as the $\mathrm{H}_{2} \mathrm{P}$ Q-band fluorescence increases. The inset shows deviations from an isosbestic point, indicating that it is not a truly bimolecular reaction.

upon addition of $\mathrm{H}_{2} \mathrm{P}$ to the QDs at low molar ratios $x=0-1$ (see the Supporting Information). This finding is in line with earlier results of a spectral (blue)shift of the $\mathrm{H}_{2} \mathrm{P}$ Q-band fluorescence upon assembly formation. ${ }^{[32]}$

\subsection{Experiments on Intrinsic PL Quenching}

For titration experiments to study the PL intensity of $\mathrm{CdSe}$ or CdSe/ZnS QDs as a function of the added amount of porphyrin molecules, all spectra were obtained within $60 \mathrm{~s}$ of the addition of the aliquot. To prove this operational procedure, we first looked into the long-term stability of the applied chemical systems, including solvent quality. In the PL emission spectra we observed intensity changes on timescales of minutes and hours. The results are described in the following sections.

\subsubsection{Time-Dependent Intrinsic PL Quenching as a Function of Solvent Impurities}

The first investigation regarded the pure master solution of the QDs. Here, after sample preparation by diluting the originally concentrated solution of QDs, we observed a change of $\mathrm{PL}$ intensity with time. This change depended on the kind of solvent, the respective purity, and the absolute concentration of QDs and surface ligands in solution. Typical examples are shown in Figure 3, left, for uncapped CdSe QDs dissolved in toluene of different grades. We clearly observed that the amount of water present in the solvent governs the stability of the PL over time, with the poorly dried toluene resulting in almost complete PL quenching within $30 \mathrm{~min}$. PL quenching of the QDs is reduced upon an increased content of TOPO in the solution (molar ratio $y=800$ ), however, the most stable PL is observed following extensive drying of the solvent, which reduces $\mathrm{PL}$ quenching considerably. The related $\mathrm{PL}$ decrease is nearly exponential at early times in all cases. The corresponding decay time constants are in the range $240-3000 \mathrm{~s}$ for the given examples. As a consequence, all quantitative experi-

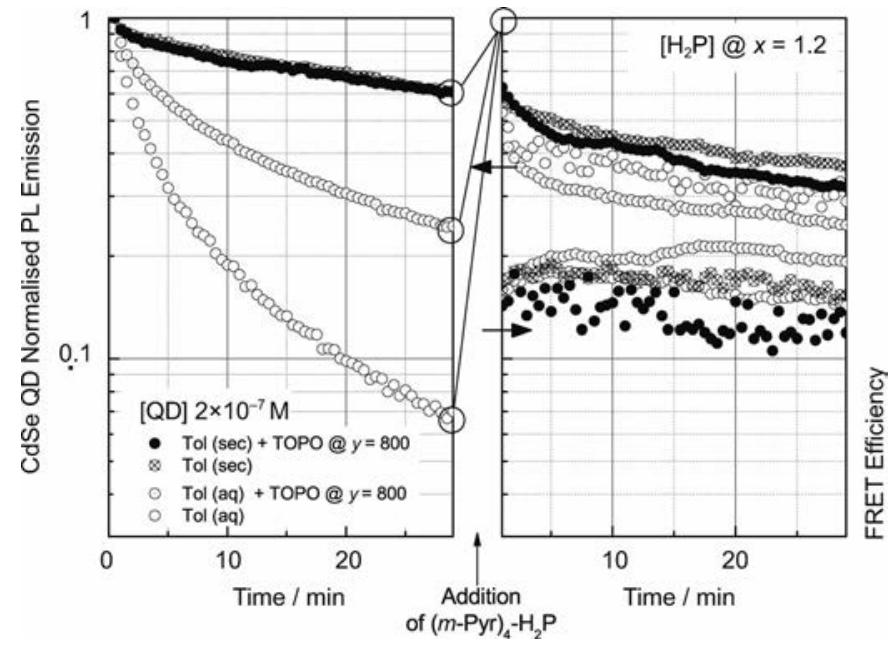

Figure 3. Influence of sample conditions on the time-dependent PL quenching of uncapped CdSe QDs in toluene. The role of different solvent qualities (left) and the influence of the addition of $(m-p y r)_{4}-\mathrm{H}_{2} \mathrm{P}$ at roughly equimolar ratios $(x=1.2)$ (right) are shown. Tol (aq) and Tol (sec) correspond to as-supplied spectroscopic grade toluene and toluene dried over molecular sieves for $48 \mathrm{~h}$, respectively. TOPO was added in a large excess (molar ratio of $y=800$ ). The PL intensities of CdSe are re-normalised with respect to the moment of addition at about $30 \mathrm{~min}$ and the time axis runs anew. The timedependent increase in the $\mathrm{H}_{2} \mathrm{P}$ fluorescence (FRET efficiency, relative units, right axis) is also shown and proves the formation of $\mathrm{QD}-\mathrm{H}_{2} \mathrm{P}$ nanoassemblies.

ments reported further below were carried out in dried toluene.

The long-term stability of aggregates in solution was investigated by adding $\mathrm{H}_{2} \mathrm{P}$ in one step at $x=1.2$ to the respective samples of uncapped CdSe QDs (see Figure 3, right). Here, for all solvent conditions, similar results were found: addition of the $\mathrm{H}_{2} \mathrm{P}$ aliquot to the master solution of QDs resulted in an immediate decrease in the $\mathrm{PL}$ intensity that was faster than our time resolution of about $60 \mathrm{~s}$. In each of the samples, the initial fast PL decrease was followed by a decrease of the PL intensities on similar timescales, but was no longer close to an exponential decay. Relative to the time behaviour of the corresponding samples before the titration step (see Figure 3, left), the decay seems to be broadly distributed (similar to a power law), which reflects the presence of different quenching processes, namely, those already inherent in the pure QD sample and those imposed by addition of the $\mathrm{H}_{2} \mathrm{P}$ resulting in assembly formation.

Figure 4 illustrates a similar scenario in terms of reaction timescales, but from the perspective of the absolute concentration of the constituents. Dried toluene was used for these experiments and absolute concentrations of QDs of $1 \times 10^{-7}$, $2 \times 10^{-7}$ and $4 \times 10^{-7} \mathrm{M}$ were prepared at the same molar ratio, $x=1.2$, as that used above. In all cases, the PL of the QDs continuously decreases; quenching is most pronounced for the most dilute solutions.

In our previous experiments, we also measured the $\mathrm{H}_{2} \mathrm{P}$ fluorescence intensity in terms of the FRET efficiency (Figures 3 and 4, right axis, lower part). ${ }^{[32]}$ It is clear that FRET increases 


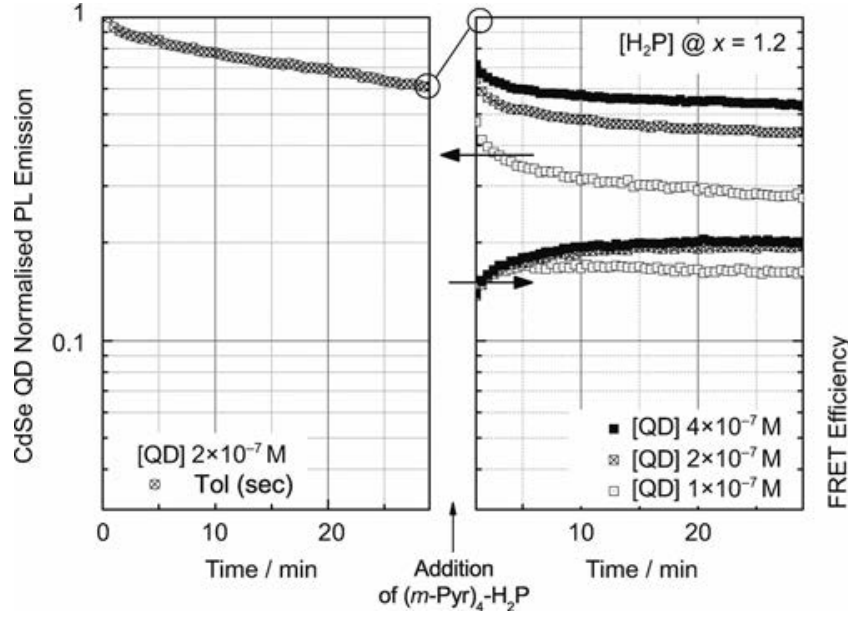

Figure 4. The role of absolute $\mathrm{QD}$ concentration $[\mathrm{QD}]$ on $\mathrm{PL}$ quenching upon the addition of $(m-\text { pyr })_{4}-\mathrm{H}_{2} \mathrm{P}$ at a roughly equimolar ratio $(x=1.2)$ (right). Again, the accompanied increase of the $\mathrm{H}_{2} \mathrm{P}$ fluorescence is also shown (right) and proves the formation of $\mathrm{QD}-\mathrm{H}_{2} \mathrm{P}$ nanoassemblies.

on a timescale of minutes before it either saturates at a constant value for several more minutes (nicely seen for [QD] = $1 \times 10^{-7} \mathrm{M}$ ) or proceeds with a slight decrease. The increase in the $\mathrm{H}_{2} \mathrm{P}$ fluorescence upon titration can be clearly explained by FRET from the QD to the adsorbed $\mathrm{H}_{2} \mathrm{P}$ molecule. It levels off as soon as it cannot compete any more with other in-growing quenching processes. This behaviour is most pronounced for the sample with the highest water content. Hence, aside from confirmation reported in earlier publications, ${ }^{[32-35]}$ FRET is a measure for the formation kinetics of $\mathrm{QD}-\mathrm{H}_{2} \mathrm{P}$ aggregates. It can be seen that PL quenching upon titration of $\mathrm{H}_{2} \mathrm{P}$ is inversely proportional to $\mathrm{QD}$ concentration. The decrease for later times is a clear result of the ongoing (independent of the $\mathrm{H}_{2} \mathrm{P}$ ) intrinsic PL quenching (Figure 4, left), which has direct feedback on FRET efficiency: in our case, neither TOPO nor $\mathrm{H}_{2} \mathrm{P}$ are protective (in contrast to refs. $[17,18,21,22]$ ), so the optical properties are mainly characterised by the particular dynamic processes at the QD surface.

\subsubsection{One-Step PL Quenching of CdSe/ZnS QDs by $\mathrm{H}_{2} P$ at Various Molar Ratios (Quasi-Static Titration)}

A third example concerns the behaviour of PL intensity as a function of the waiting time after one fixed titration step. In this experiment, the long-term stability of the PL of core-shell CdSe/ZnS QDs was investigated at various $\mathrm{H}_{2} \mathrm{P}$ concentrations (Figure 5). Here, samples at different molar ratios, $x$, were prepared in one single titration step, which resulted in PL quenching similar to that discussed above for the uncapped QDs. The data for this quasi-static titration are presented as the ratio of $I(x)$ (the intensity after titration) to $I_{0}$ (the intensity just before the first titration event). It can be clearly seen that PL quenching increases with waiting time. This effect is most pronounced for high molar ratios indicated by the additional intensity loss of $15 \%$ over $3.5 \mathrm{~h}$ at $x=11$, whereas solutions at molar ratios of $x=0-6$ remain with an acceptable additional intensity loss

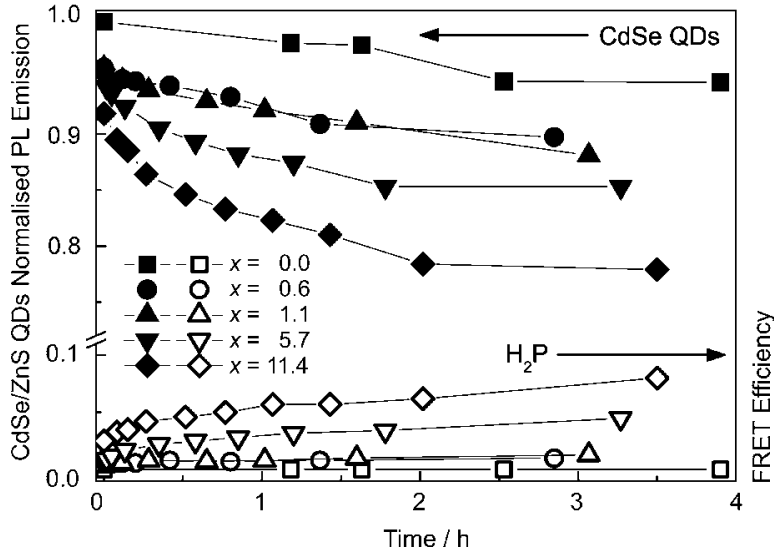

Figure 5. Long-time development of the PL of CdSe/ZnS QDs and FRET efficiency in presence of $(m \text {-Pyr })_{4}-\mathrm{H}_{2} \mathrm{P}$ molecules for $\mathrm{QD}-\mathrm{H}_{2} \mathrm{P}$ assemblies at different molar ratios. The values on the left axis, $I(x) / I_{0}$, were normalised to the $\mathrm{PL}$ of the individual sample prior to titration. A comparison of $\mathrm{PL}$ quenching with FRET build up over time shows that the QD- $\mathrm{H}_{2} \mathrm{P}$ assembly formation has multiple (at least three) macroscopic timescales.

in the range of $5-8 \%$ over $3.5 \mathrm{~h}$. The absolute efficiency of quenching increases with $x$, although again some quenching is present in the absence of $\mathrm{H}_{2} \mathrm{P}$.

\subsection{PL Quenching as a Function of Molar Ratio, Absolute QD Concentration, and Size}

Up to this point, the results presented show the applicability of steady-state fluorescence titration as a quantitative analysis tool for the formation of aggregates from semiconductor QDs and functionalised porphyrin molecules in solution. In the following sections, we discuss some particular factors that influence the PL quenching of CdSe and CdSe/ZnS QDs in QD- $\mathrm{H}_{2} \mathrm{P}$ aggregates.

\subsubsection{Influence of Absolute Concentration on PL Quenching of CdSe QDs}

Figure 6 shows the results of PL quenching experiments for uncapped CdSe QDs with (m-Pyr) $)_{4}-\mathrm{H}_{2} \mathrm{P}$ at absolute concentrations ranging from $4 \times 10^{-8}$ to $4 \times 10^{-7} \mathrm{M}$. Figure $6 \mathrm{~A}$ depicts the normalised PL intensity $I(x) / I_{0}$, in which $I_{0}$ is the PL intensity of the QDs without $\mathrm{H}_{2} \mathrm{P}$ and $I(x)$ is the (quenched) $\mathrm{PL}$ intensity in presence of $\mathrm{H}_{2} \mathrm{P}$ molecules. Figure $6 \mathrm{~B}$ shows the respective Stern-Volmer plots of the reciprocal PL intensity ratio $I_{0} / I(x)$. In the main parts of Figure $6 \mathrm{~A}$ and $\mathrm{B}$, the abscissae correspond to the absolute quencher concentration $\left[\mathrm{H}_{2} \mathrm{P}\right]$ (as traditional Stern-Volmer formalism), while the abscissae in the insets correspond to the molar ratio $x$. It can be seen that the ratio $I_{0} / I(x)$ increases linearly with $\left[\mathrm{H}_{2} \mathrm{P}\right]$ at low concentrations, but with an increment different for each absolute concentration. At higher $\mathrm{H}_{2} \mathrm{P}$ concentrations, the ratio $I_{\mathrm{o}} / /(x)$ becomes smaller with absolute quencher concentration (as seen in Figure 6B), but still increases linearly with $x$, where the slope of the increase is constant for all curves (as seen in the plot over the molar ratio in the inset of Figure $6 \mathrm{~B}$ ). For $[\mathrm{QD}]=4 \times 10^{-8} \mathrm{M}$, due to sensitivity 

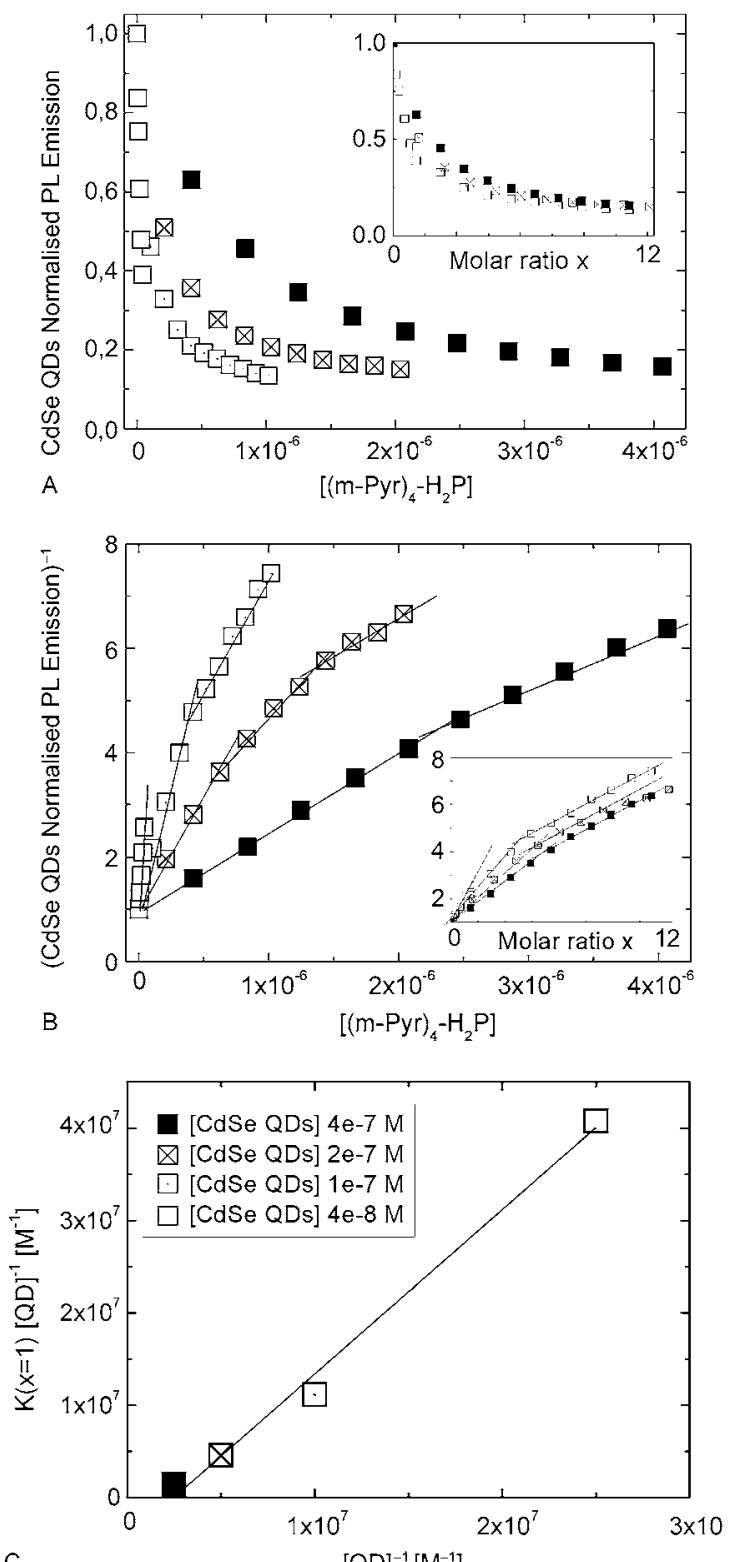

C

$[\mathrm{QD}]^{-1}\left[\mathrm{M}^{-1}\right]$

Figure 6. A) $\mathrm{PL}$ quenching, $I\left(\left[\mathrm{H}_{2} \mathrm{P}\right]\right) / I_{0}$, of $\mathrm{CdSe} \mathrm{QDs}$ as a function of the concentration of $(m-P y r)_{4}-\mathrm{H}_{2} \mathrm{P}$ quencher molecules for titration experiments at four different absolute concentrations of [QD]. B) Stern-Volmer plot derived from A), showing $I_{0} / /\left(\left[(m-P y r)_{4}-\mathrm{H}_{2} \mathrm{P}\right]\right)$. Here, the data points can be approximated by linear functions with two different slopes. C) The slope $K(x=1)$ (low-molar-ratio regime) evaluated according to Equation (1). $K(x=1)$ is independent of $[Q D]$, which means that the QD PL quenching processes are identical. The insets in $A$ ) and $B$ ) show the same PL quenching as a function of the molar ratio of $x=\left[\mathrm{H}_{2} \mathrm{P}\right] /[\mathrm{QD}]$, indicating that the same is true for quenching processes at higher molar ratios.

reasons, we could not follow the behaviour over the total concentration range. Based on the analysis of the Stern-Volmer plots, Figure $6 \mathrm{C}$ reveals the linear relationship between the quenching constant $K$ [determined for small molar ratios according to Eq. (2) below] and the reciprocal absolute concentration $[Q D]^{-1}$, respectively. The linear dependence indicates that the underlying quenching processes have a common nature for all absolute concentrations.
Experiments on PL quenching of CdSe QDs at variable absolute QD concentration, as shown in Figure 6, confirm the assumption of dynamically controlled quenching below and above a critical molar ratio, $x_{c}$ : both below and above this value there is linearity in the Stern-Volmer plot in any curve. We conclude that, for the QD-dye assemblies, the formation dynamics change above a critical value of the quencher concentration. Above this value additional dynamic constraints, such as ligand dynamics, are to create new attachment sites which influence the PL of the QD in addition to the immediate assembly formation.

\subsubsection{Influence of QD Size on PL Quenching of CdSe/ZnS QDs by $\mathrm{H}_{2} \mathrm{P}$}

As an inevitable basis for the discussions in this paper, we present in Figure $7 \mathrm{~A}$ PL quenching as a function of the molar ratio of different types of QDs at fixed $Q D$ concentrations $\left(10^{-7} \mathrm{M}\right)$ from an earlier publication. ${ }^{[33]}$ For the analysis of the $\mathrm{PL}$ quenching as a function of the number of $\mathrm{H}_{2} \mathrm{P}$ molecules per QD for various QD sizes, the well-known Stern-Volmer formalism ${ }^{[38,39]}$ was modified. In our more generalised approach, the luminescence quenching can be described by Equation (1):
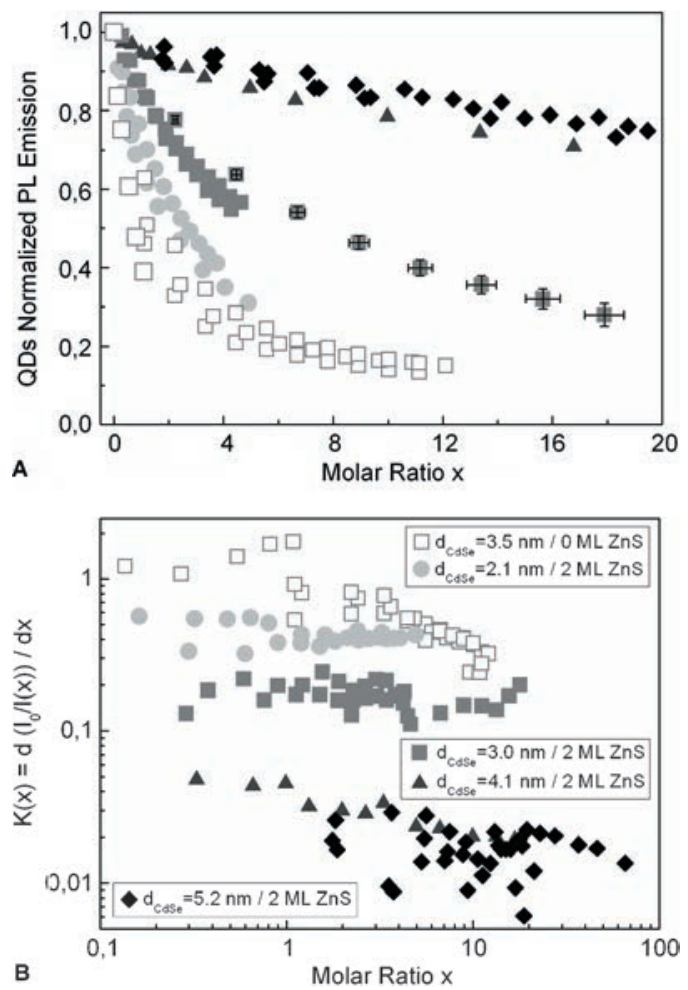

Figure 7. A) PL quenching, $I(x) / I_{0}$, of both uncapped CdSe QDs (open markers) and capped CdSe/ZnS QDs (solid markers) upon titration of $(m-\mathrm{Pyr})_{4}-\mathrm{H}_{2} \mathrm{P}$ as a function of the molar ratio $x=\left[\mathrm{H}_{2} \mathrm{P}\right] /[\mathrm{QD}]$. B) Stern-Volmer function $K(x)$ calculated, according to Equation (1), for all data points in A). The double logarithmic plot shows the clear dependence of PL quenching on QD size. $\mathrm{ML}=$ monolayers. 
$\frac{I_{0}}{l}=1+\int_{0}^{\infty} K(x) \mathrm{d} x$

In Equation (1), $I(x)$ and $I_{0}$ represent the PL emission intensity of the QD in the presence and absence of quencher, respectively. In this approach, the Stern-Volmer function, $K(x)$, depends explicitly on the molar ratio, $x$, of the molar concentrations of the QDs and (m-Pyr $)_{4}-\mathrm{H}_{2} \mathrm{P}$ and is expressed as the first derivative of the experimental data plotted in a Stern-Volmer representation. Furthermore, the Stern-Volmer "constant" (now as a function of $x), K(x)$, can be written as Equation (2):

$K(x)=k_{\mathrm{q}}(x) \tau_{0}$

in which $k_{\mathrm{q}}$ corresponds to the total quenching rate induced by the number of quencher molecules actually present (depending on molar ratio $x$ ) and $\tau_{0}$ corresponds to the intrinsic $\mathrm{PL}$ lifetime of the QD in absence of quencher molecules. Fluorescence lifetimes are known from measurements ${ }^{[33]}$ or can be extrapolated from the literature. ${ }^{[40]}$ Note that, in this generalised Stern-Volmer formalism, $K$ is non-dimensional value. As known from our previous study, ${ }^{[33]} I_{\mathrm{o}} / I(x)$ does not show a linear correlation with $x$ over the total $\mathrm{H}_{2} \mathrm{P}$ concentration range. This can be more easily seen in Figure 7B where we have plotted $K(x)$ on a double logarithmic scale. It can be seen that $K(x)$ is indeed initially constant, but becomes smaller around a critical molar ratio (which we call $x_{c}$ ). Additionally, this critical molar ratio, $x_{c}$ increases more or less systematically with the diameter of the QD. As discussed later, a non-constant, $K(x)$, indicates a deviation from a normal Stern-Volmer relationship in the case of a truly bimolecular reaction. ${ }^{[3]}$

The overall interpretation of the above findings is that $\mathrm{PL}$ quenching by titration with $\mathrm{H}_{2} \mathrm{P}$ occurs in two steps. 1) Immediately after titration, $\mathrm{QD}-\mathrm{H}_{2} \mathrm{P}$ nanoassemblies are effectively formed, which results in both PL quenching of the QD PL and FRET to the adsorbed dye molecules. ${ }^{[32]}$ 2) More dye molecules become attached during the waiting time. However, increased $\mathrm{PL}$ quenching and FRET are not exactly on the same quantitative level. Clearly the titration step also favours other quenching mechanisms than those related to FRET. ${ }^{[33]}$ This makes us believe that the dynamics initiated by a single titration step are not only due to the added $\mathrm{H}_{2} \mathrm{P}$ molecules themselves, but also to a local change in the ligand shell on the QD surface upon assembly formation. This argumentation is in line with the finding that dilution of a QD solution reduces the average coverage of the QDs with TOPO, ${ }^{[36,37]}$ giving rise to both an intrinsic reduction of the QD PL emission, as reported by Munro et al., ${ }^{[12]}$ as well as to increased accessibility of the QD surface to the quencher molecules.

Aside from the formation processes, the role of the solvents used and the stability of the $\mathrm{H}_{2} \mathrm{P}$ molecules attached to the ligand shell were investigated, both in solution by competitive aggregate formation in comparison to multiporphyrin complexes $^{[41-43]}$ and in a polymer film deposited on a silicon wafer $^{[44]}$ (see the Supporting Information). There are two basic results: 1) ligand coverage, and thus, the number of accessible attachment sites are controlled by the solvent polarity and 2) attachment of $\mathrm{H}_{2} \mathrm{P}$ does not result in a permanent change in the physical properties of the QDs.

\section{Discussion}

Recent experiments have shown that it is possible to form nanoassemblies with pyridyl-functionalised dyes that contain CdSe QDs as building blocks through self-assembly principles. ${ }^{[32-37,41,43]}$ We showed for porphyrins, ${ }^{[32-35]}$ and also for perylene diimide (PDI) dyes, ${ }^{[36,37]}$ that the lone pair orbitals of the pyridyl groups were the essential keys to attach organic moieties to the CdSe or ZnS QD surface by forming a coordinative chemical bond with the orbitals of outer $\mathrm{Cd}^{2+}$ or $\mathrm{Zn}^{2+}$ ions. ${ }^{[32,33]}$ Recent calculations with respect to the bonding conditions of ligands ${ }^{[45]}$ have shown that the bond strength for the ligands can be described in most cases by electrostatic interactions between the partial charges of the respective surface ions and the ones of the ligand anchoring group.

We assume that similar conditions also hold for the formation of nanoassemblies by pyridyl anchoring groups, since $\mathrm{H}_{2} \mathrm{P}$ becomes attached to the QD surface in a nearly perpendicular orientation $^{[32,37]}$ (see Figure $1 \mathrm{~A}$ ). The partial charge of pyridine is about -0.747 at the nitrogen atom. ${ }^{[46]}$ Since the bond is electrostatic, the binding strength has to be corrected for the dielectric properties of the solvent. ${ }^{[4]}$ Nevertheless, pyridine anchor groups should be able to establish a nanoassembly.

A necessary condition for such a formation is the fact that geometric constraints allow effective bond formation. An example is that, for steric reasons, ortho-pyridyl-substituted porphyrins are not subject to assembly formation, contrary to para- or meta-substituted ones. ${ }^{[32]}$ In all respective $\mathrm{PL}$ quenching experiments, we observed that assembly formation was accompanied by FRET and non-FRET processes, ${ }^{[32-36]}$ but we could exclude photoinduced charge transfer. ${ }^{[32,36]}$ While the formation of assemblies is manifested by PL quenching through a uniquely identified mechanism ${ }^{[33]}$ in combination with FRET (enhancement of dye acceptor fluorescence), ${ }^{[32]}$ three issues remain open: 1) the identification of the average number of dye molecules attached to the QD surface, 2) the formation dynamics of such nanoassemblies and 3 ) the closely related dynamics of ligands. In each case, the dynamics of assembly formation is closely related to ligand dynamics, since the dye molecule either has to find freely accessible sites on the QD surface or has to partly replace the existing ligand shell.

Reports on ligand dynamics mostly deal with uncapped CdSe QDs, ${ }^{[10-12,45,47-50]}$ since the effects on PL quenching following ligand dynamics are much more pronounced for uncapped than for capped systems, for example, CdSe/ZnS QDs. We present results on capped $\mathrm{CdSe} / \mathrm{ZnS}$ QDs, which generally exhibit much less variation in PL intensities. Moreover, the application of dyes offers the possibility of investigating surface attachments on an extremely low concentration level that is not accessible by conventional experiments on ligand dynamics. Additionally, as shown experimentally ${ }^{[33]}$ and theoretically, ${ }^{[51]}$ the use of dyes allows investigations into the microscopic aspects of the quenching process itself. 


\subsection{Proof of QD-Dye Assembly Formation}

The determination of the number of dye molecules per QD over the course of titration experiments is difficult, since the overall PL quenching depends both on the (prior unknown) quenching efficiency and on the number of dye molecules per QD. To separate these two effects, one needs the independent identification of the number of attached $\mathrm{H}_{2} \mathrm{P}$ molecules merely from spectroscopic means.

For PDI dyes, we recently successfully addressed this question on at the single-molecule level. ${ }^{[36,37]}$ By comparing $\mathrm{PL}$ quenching experiments for single molecules and ensembles, they showed that, on average $0.24 \mathrm{PDI}$ dye molecules assemble on a CdSe/ZnS QD when starting with an equimolar (i.e., $1: 1)$ ratio in toluene, however, no spectral information on the QD-PDI assembly information was available. For QD- $\mathrm{H}_{2} \mathrm{P}$ assembly formation, the spectra show that only a small part of $\mathrm{H}_{2} \mathrm{P}$ added during the titration procedure becomes attached to the surface, as indicated by the missing isosbestic point (Figure $2 \mathrm{~A}$ ). Herein, on one hand, we did not observe any noticeable spectral shifts in the PL of the QDs upon assembly formation (Figure 2). On the other hand, the $\mathrm{H}_{2} \mathrm{P}$ fluorescence exhibited spectral blueshifts of the $Q$ band ${ }^{[32]}$ as well as redshifts of both $\mathrm{Q}$ and Soret band absorptions (see the Supporting Information).

There are several possible explanations for the observed spectral shifts. In principle, the spectral properties depend on several factors, such as molecular geometry, particle surface composition, overall dielectric function and polarisation effects through the meso-pyridyl rings upon attachment to the QD surface. A redshift (both for absorption and emission) is expected in relation to an increase in the average dielectric constant, which is approximately $\varepsilon=9.7$ for CdSe and $\varepsilon=2.4$ for toluene. On the contrary, the Q-band emission exhibits a blueshift that cannot be explained by a dielectric model. The Stokes shift between Q-band absorption and emission becomes smaller upon assembly formation. At the same time, the Q-band fluorescence of $\mathrm{H}_{2} \mathrm{P}$ at high energy $(653 \mathrm{~nm})$ is reduced relative to the purely vibrational one (at $720 \mathrm{~nm}$ ), which indicates that the Franck-Condon factor changes upon assembly formation. It is known that the Q-bands in $\mathrm{H}_{2} \mathrm{P}$ correspond to symmetry-forbidden transitions ${ }^{[52]}$ that become partly allowed by distortion of the $\mathrm{H}_{2} \mathrm{P}$ ring upon attachment to the QD. This induces an increased Stokes shift and an increase of the formerly forbidden (vibration-less) transition at high energy. We assume that the same processes hold for the situation of $\mathrm{H}_{2} \mathrm{P}$ in toluene. The assembly formation clearly restores the intrinsic $\mathrm{H}_{2} \mathrm{P}$ symmetry (a planar $\mathrm{H}_{2} \mathrm{P}$ ring), which has a symmetry-forbidden optical transition. Planarity is probably enforced when the $\mathrm{H}_{2} \mathrm{P}$ molecules coordinate to the QD surface and interact with the closely packed TOPO shell in the vicinity. For PDI dyes, supporting this hypothesis, we performed singlemolecule experiments that showed the complex structure of these dyes, on average, changed upon surface attachment in favour of a flatter structure, but with reduced flexibility. ${ }^{[36,37]}$

Of course, this picture is certainly oversimplified, since different magnitudes in the spectral shifts for various QD assemblies were observed without finding clear systematic behaviour for all of the systems. Clearly, the shifts depend on the type of QD and decrease with the molar ratio $x$ (see the Supporting Information). For large molar ratios, the shifts vanish and one can conclude that the spectroscopic properties are, on average, determined by non-assembled $\mathrm{H}_{2} \mathrm{P}$ molecules. The critical molar ratio at which this happens is in the order of $x_{c}=1-10$ and increases with increasing QD size.

We conclude that spectral shifts prove assembly formation immediately after the first titration step at $x<x_{c}$ conducted in our case for molar ratios as low as $x \approx 0.1$. At molar ratios of $x>x_{c}$ (with $x_{c}=1-10$, depending on QD size, see below), the porphyrins become less and less complexed. From studies on QD-PDI nanoassemblies, we know from single-molecule and single-QD experiments that one dye molecule causes PL quenching for each QD that is, at most, twice as strong as that in solution at $x=1 .^{[36,37]}$ From this observation, we deduced that only a smaller number of dyes (about one in four) ${ }^{[36]}$ attached to the QD surface at comparable molar ratios in solution. For $\mathrm{QD}-\mathrm{H}_{2} \mathrm{P}$ nanoassemblies, fluorescence lifetime measurements support a complexation efficiency that is in the same order of magnitude: for individual molecules of $(m-\mathrm{Pyr})_{n} \mathrm{H}_{2} \mathrm{P}$, fluorescence decay in the absence of QDs in toluene is $8.4 \mathrm{~ns}^{[52,53]}$ For the first titration step at $x \approx 0.25$, the mono-exponential lifetime increases to $11 \mathrm{~ns}$ due to FRET from the long-lived CdSe/ZnS QDs. ${ }^{[54]}$ This fact implies that, at these low molar ratios, most of the $\mathrm{H}_{2} \mathrm{P}$ molecules are attached to a $\mathrm{QD}$, whereas the complexation efficiency reduces considerably when the molar ratio is increased. This discussion is in line with results on the shift of the $\mathrm{H}_{2} \mathrm{P}$ Soret band (see the Supporting Information). All of these experiments show that one attached dye molecule can already quench the PL intensity of a QD considerably. Further information on the number of $\mathrm{H}_{2} \mathrm{P}$ per QD is presented below from an analysis of the PL quenching, following the detailed Stern-Volmer description given in Section 3.2.

\subsection{Dye Attachment Dynamics}

Our starting working hypothesis is that QD-dye assemblies are formed in a dynamic equilibrium between attachment and detachment of dye molecules to the QD surface. Time-dependent $\mathrm{PL}$ experiments on QD- $\mathrm{H}_{2} \mathrm{P}$ assembly formation (Figure 3 ) show that the time to reach the initial equilibrium is faster than our experimental observation time of about $60 \mathrm{~s}$. From this we conclude that there should be some free attachment sites on the QD surface, since ligand replacement would be on timescales of minutes or even longer. From the size-dependent PL quenching experiments (Figure 7), we further conclude that assembly formation can be tentatively divided into a lowand high-molar-ratio range, which is separated by a "critical" molar ratio, $x_{c}$. Above $x_{c}$ the probability of attaching dye molecules to the QD surfaces is considerably reduced. By applying our generalised Stern-Volmer analysis of the QD PL quenching, ${ }^{[33]}$ and assuming a piecewise linear correlation of $I(x)=I_{i}=\operatorname{const}\left(x_{i}<x<x_{i+1}\right)$ after the ith titration step, Equation (1) can be written as Equation (3): 
$\frac{I_{o}}{I_{i}}=1+K\left(x_{i}\right) x_{i}$

In Equation (3), $I_{0}=I(x=0)$ is the PL intensity at molar ratio $x=0$, while $I_{i}=I\left(x_{i}\right)$ is the intensity at a given molar ratio $x$ after the $i$ th titration step. For the sake of clarity, the index $i$ is omitted in the following discussion. In our generalised approach, the Stern-Volmer quenching constant $K$ depends on $x$ if, for example, dye attachment is limited by the number of accessible sites, $n_{\max }$ on a QD surface. This number can also vary according to particular conditions, for example, if a rearrangement of the ligand shell or the surface topography ${ }^{[26]}$ has to happen to allow for further dye attachment.

Now let us assume that, at low $x$, the QDs have free abundant or easily accessible attachment sites available for assembly formation with dye molecules. At these low concentrations, quenching occurs for stable assemblies and it is very similar to doping-dependent transfer processes in molecular crystals. ${ }^{[55]}$ In that low-molar-ratio range, $K_{x<x_{c}}$ is independent of $x$ and can be described [according to Eq. (2)] by $K_{x<x_{c}}=K_{c}^{r}=k_{\mathrm{q}} \tau_{0}$, in which $k_{\mathrm{q}}$ corresponds to the effective quenching rate and $\tau_{o}$ corresponds to the intrinsic excited-state lifetime of the QD PL. Note that in our generalised approach, contrary to the standard Stern-Volmer formalism, $K$ is dimensionless (instead of $\mathrm{M}^{-1}$ ) and $k_{\mathrm{q}}$ carries the unit $\mathrm{ns}^{-1}$ instead of $\mathrm{M}^{-1} \mathrm{~ns}^{-1}$ like other rate constants: both variables are referred to the molar ratio $x$ (quencher molecules per fluorophore or QD) instead of the quencher concentration. This in no way violates the validity of the approach because even standard Stern-Volmer formalism often deals with a reference concentration of fluorophores (see ref. [39], p. 149).

In previous publications, we have shown that $k_{\mathrm{q}}$ describes both FRET and non-FRET quenching mechanisms. ${ }^{[32,33]}$ For molecules of $\mathrm{H}_{2} \mathrm{P}^{[33]}$ and $\mathrm{PDI}^{[36]}$, the non-FRET quenching mechanisms were identified to be dominant. Having carefully extracted the non-FRET contributions from total PL quenching, we recently suggested a rate constant for the non-FRET quenching, which is given by Equation (4): ${ }^{[33,51]}$

$k_{\mathrm{q}}=C \Psi^{2}$

in which $\Psi^{2}$ corresponds to the probability of the electron wave function of the exciton at the site of the dye attachment, that is, at the QD surface. Here, the variable $C$ includes all static and/or dynamic effects of the attachment process itself. This approximation makes use of the fact that the exciton wave function extends beyond the QD core (quantum-size effect), and gives rise to quenching processes mediated by, for example, dye attachment. ${ }^{[33]}$

The above generalised formalism allows a quantitative analysis of the Stern-Volmer plots to reveal size-dependent PL quenching. ${ }^{[33]}$ For Figure $7 \mathrm{~B}$, we observe that $K$ clearly depends on the molar ratio $x$ and is nearly constant, again up to a critical concentration, $x_{c}$. However, both the absolute value of $K$ and $x_{c}$ depend on the size of the QDs. For the low-molar-ratio range $x<x_{c}$, the dependence of the magnitude of $K_{x<x_{c}}=K_{c}^{r}$ on size is in agreement with earlier results, namely, that $K_{c}^{r}$ is related to $\Psi^{2}$ at the place of dye attachment. ${ }^{[33]}$

It should be noted that, in previous publications, ${ }^{[32-35]}$ the origin of the observed $x_{c}$ remains unknown. In the following discussion, we show that, at this point, the QD surface is far from being totally covered with dye molecules, but we concentrate on the fact that this value represents a landmark where the major processes responsible for assembly formation change. With our generalised Stern-Volmer formalism, the results of Figure 7B allow the determination of $x_{c}$ and the modified equilibrium constant $K_{x>x_{c}}=K_{c}^{\mathrm{d}}$ for the high-molar-ratio range. In Figure $8 \mathrm{~A}$, for the same absolute QD concentration, we have plotted all $x_{c}$ values (which should be related to the maximum number of freely accessible binding sites $n_{\max }$ ) as a function of the QD surface area calculated in spherical approximation. The plot reveals linear behaviour and we conclude that the number of freely accessible sites is proportional to the surface area of the respective QD. From the slope of the linear relationship, we determine that (normalised by the QD surface unit) $n_{0} \leq(0.032 \pm 0.006) \mathrm{H}_{2} \mathrm{P}$ molecules per $\mathrm{nm}$ can freely attach the CdSe QD. Hence, the average number of attached molecules $n_{\max }$ corresponds to the QD size through $x_{c}$.
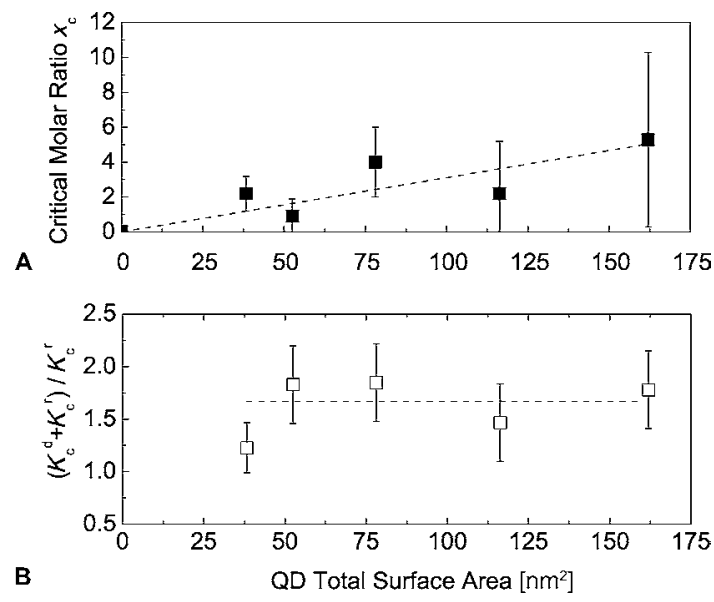

Figure 8. A) Critical molar ratio $x_{c}$ for various types of QDs as a function of the QD surface area $4 \pi(R+D)^{2}$ calculated from the radius $R$ of the CdSe core and eventually the thickness $D$ of the $\mathrm{ZnS}$ shell. B) The ratio $\left(K_{\mathrm{c}}^{\mathrm{d}}+K_{\mathrm{c}}^{\mathrm{r}}\right) / K_{\mathrm{c}}^{\mathrm{r}}$ as a function of QD surface area.

According to this linear relationship, $n_{\max }$ is between 0.8 (for the smallest species: uncapped CdSe QDs with a core diameter of $3.0 \mathrm{~nm}$ ) and 5.3 (for the largest CdSe/ZnS QDs with a core diameter $5.2 \mathrm{~nm}$ ) $\mathrm{H}_{2} \mathrm{P}$ molecules per QD. This is only an upper limit and the real number of attached dye molecules $n$ may be by far smaller than $n_{\max }$ because there is not a direct measure for this number. Again, according to the experiments on PDI, this number may be a factor of up to five smaller than that of $n_{\max }{ }^{[36,37]}$ Even if $n$ can be estimated from the corresponding absorption coefficients for QD and dye after precipitation and re-dissolution of the nanoassemblies, ${ }^{[23]}$ competitive attachment-detachment processes may take place. The important conclusion from Figure $8 \mathrm{~A}$ is that $n$ is almost linearly related to 
the QD surface area. However, from numerical considerations, it is reasonable to ask why a surface area of $30 \mathrm{~nm}^{2}$ (representing a QD of $3.2 \mathrm{~nm}$ diameter) is required to effectively host a single dye molecule (estimated from a maximum occupied area of $1.2 \mathrm{~nm}^{2}$ for $\left.\mathrm{H}_{2} \mathrm{P}\right){ }^{\left[{ }^{[32]}\right.}$ Our interpretation is that only selected parts of the QD surface area allow immediate attachment of the dye molecules because it is inhomogeneous with respect to ligand dynamics. This argument is clear from theoretical investigations into the growth mechanisms of semiconductor nanocrystals, leading to facet structures, ${ }^{[56,57]}$ as well as experiments about a complex optical transition moment attributed to strain within the crystal or anisotropic growth of QDs during synthesis ${ }^{[58]}$ (see simplified sketch in Figure $1 \mathrm{~B}$ ). The relationship between specific surface facets and ligand binding have been discussed recently through numerical simulations by Voznyy. ${ }^{[26]}$ In the view of this picture, we see that particular crystal facets of the QDs or even the edges and corners of these facets have different chemical properties; this is relevant for immediate attachment of the dye molecules in solution: only sub-areas with a particular structure contribute to immediate attachment without significant competition with the original ligand (TOPO). When the attachment sites in these areas are filled (in our picture, this happens around $x=x_{c}$ ), further PL quenching is controlled by more complex processes that are based on competition between the dye molecules and the original ligands (TOPO) to attach to the QD surface. In the present case, the immediately accessible part of the QD surface area is the ratio between the areas of the $\mathrm{H}_{2} \mathrm{P}$ quencher molecule $\left(1.2 \mathrm{~nm}^{2}\right)$ and the "effective area" to host one quencher molecule on a QD surface (around $30 \mathrm{~nm}^{2}$ ); this value can be deduced by PL quenching experiments. Recently, we have concluded that, when assuming a relationship between PL intensity and the TOPO coverage of a $Q D$, one dye molecule must replace a factor of 10 more TOPO ligands, as expected from volume considerations, when taking a linear relationship between PL intensity and ligand coverage into account. ${ }^{[36,37]}$ However, from the present results, we have to admit that PL intensity is not a linear but (at least) a bi-linear function of TOPO coverage, which corresponds to (at least) two classes of ligand attachment sites on a QD surface. This finding has consequences for the efficiency of non-FRET PL quenching processes: dye-induced non-FRET quenching relates to ligand replacement that is different for different chemical properties of these sites. In other words, our experiments show that it is possible to probe the interface dynamics of QDs with dye molecules in solution, even from ensemble experiments, by observing that a simplified picture of a spherical QD does not fully explain the details. This is intriguing despite the clear correlation between initial quenching for small $x$ and the amplitude of the exciton wave function at the QD surface.

In light of these considerations, it may be clear that around $x_{c}$ the Stern-Volmer quenching constant $K$ becomes dependent on $x$ itself (Figure 7B). After being constant, $x_{c}$ marks the turning point to a lower value with increasing molar ratio $x$. Since there is experimental evidence, let us assume that $K$ levels off for $x \gg x_{c}$. To get further along with the quantitative analysis, we define $K_{x \rightarrow 0}=K_{\mathrm{c}}^{\mathrm{r}}$ and $K_{x \rightarrow \infty}=K_{\mathrm{c}}^{\mathrm{d}}$ below and above the critical molar ratio $x_{c}$, respectively. Although for large $x$ the saturation to a constant value is not as pronounced as for low $x$, this conjecture holds roughly because the deviations can be attributed to the long-term quenching mechanisms discussed above (Figure 5). Based on the generalised Stern-Volmer approach, the data points from Figure 7B can be fitted with a step function (see the Supporting Information). Because this step requires binning of the data, the modified plot from Figure $7 \mathrm{~B}$ is shown in Figure 9. While the determination of $K_{c}^{\mathrm{r}}$ is quite reliable (since it is nearly constant in the low concentration range), $K_{c}^{\mathrm{d}}$ is less well defined.

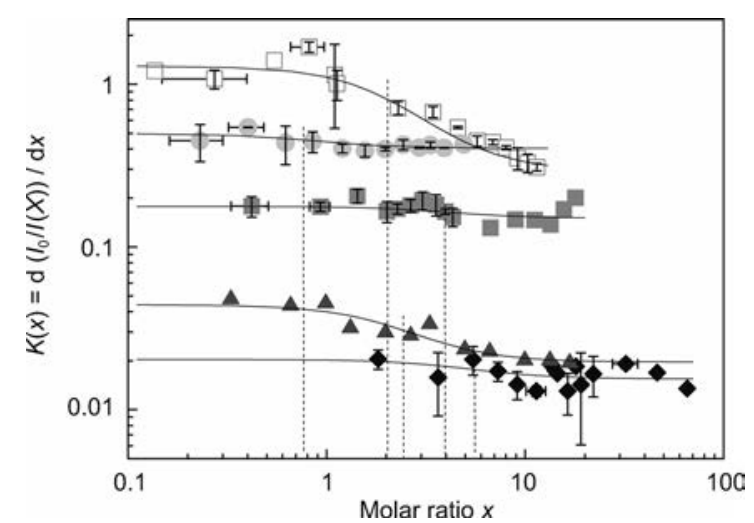

Figure 9. The curves shows the Stern-Volmer function $K(x)$ calculated for a binned set of data points from Figure 7B fitted with a step function levelling from $K_{x<x c}=K_{\mathrm{c}}^{\mathrm{r}}$ to $K_{x>x c}=K_{\mathrm{c}}^{\mathrm{d}}$. The dashed vertical lines indicate $x_{\mathrm{c}}$ for each sample of QDs determined from the fit. See legend in Figure $7 \mathrm{~B}$.

When plotting $\left(K_{\mathrm{c}}^{\mathrm{d}}+K_{\mathrm{c}}^{\mathrm{r}}\right) / K_{\mathrm{c}}^{\mathrm{r}}$ as a function of QD surface area (see Figure $8 \mathrm{~B}$ ), we obtain a nearly constant ratio between $K_{c}^{\mathrm{d}}$ and $K_{c}^{r}$ (slope of $1.67 \pm 0.09$ ). From this observation, we conclude that the number of sites accessible, for example, by reconstruction of the ligand shell ${ }^{[26]}$ is also related to the surface area. Hence there are reasonable arguments for surface attachment taking place in two steps: 1) direct attachment and 2) attachment following surface or surface-ligand reorganisation. The crossover between these two routes (around $x_{c}$ ) depends on the total QD surface area and hence also on the QD size.

For a quantitative comparison of equilibrium constants with the literature we extend our model from reference [33]. In standard literature on the Stern-Volmer formalism, ${ }^{[38,39]}$ the equilibrium constant $K_{c}^{\mathrm{X}}$ of quencher $\mathrm{X}$ with respect to fluorophore $A$ in dynamic equilibrium is given by Equation (5):

$K_{\mathrm{c}}^{\mathrm{X}}=\frac{[\mathrm{AX}]}{[\mathrm{A}][\mathrm{X}]}$

In our case, A stands for the QDs and $\mathrm{X}$ for the $\mathrm{H}_{2} \mathrm{P}$ molecules. Following discussions given in reference [33], we introduce a variable parameter $B$ according to Equation (6):

$C=B K_{c}$ 
in which $B$ describes all of the mechanistic details of the PL quenching process. In Equation (6), $K_{c}$ is a modified dynamic equilibrium constant that is related to the standard equilibrium constant by normalisation with the absolute concentration of the fluorophore, that is, $K_{\mathrm{c}}^{\mathrm{X}}=K_{\mathrm{c}}[\mathrm{QD}]^{-1}$. Note that $K_{\mathrm{c}}$ takes the number $n$ of dye molecules actually complexed to a QD into account and may differ from what is expected according to the adjusted molar ratio $x$.

We can now consider the situation for low and high molar ratios separately. For low molar ratios, the $\mathrm{PL}$ quenching rate, $k_{\mathrm{q}}$, can be plotted versus $\Psi^{2} K_{\mathrm{c}}^{\mathrm{r}}[\mathrm{QD}]^{-1}$ (Figure 9). Here we apply Equation (4) and take $k_{\mathrm{q}}$ and $\Psi^{2}$ from references [33] and [51] (see Tables A.I and A.II in the Supporting Information). Now constant $B$ can be determined from the slope in Equation (7):

$k_{\mathrm{q}}=B K_{\mathrm{c}}^{\mathrm{r}}[\mathrm{QD}]^{-1} \Psi^{2}$

For CdSe/ZnS QDs of various sizes, the graph in Figure $10 \mathrm{~A}$ exhibits an almost linear relationship, which means that the microscopic quenching mechanism is clearly the same for the
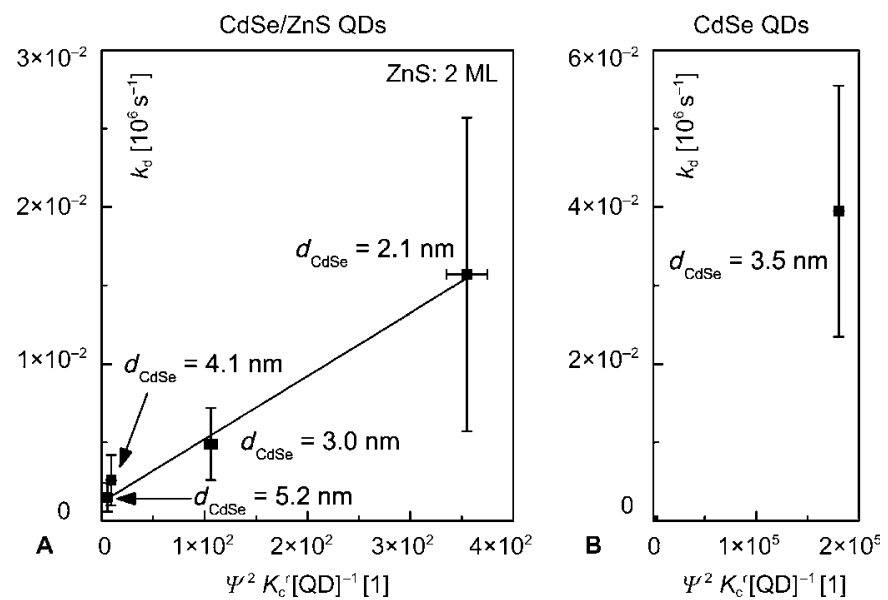

Figure 10. Quenching rate, $k_{\mathrm{q}}$, as a function of $\Psi^{2}(R+D) K_{\mathrm{c}}^{\mathrm{r}}[\mathrm{QD}]^{-1}$ for A) CdSe/ $\mathrm{ZnS}$ and B) CdSe QDs. The almost linear relationship in A) means that the microscopic quenching mechanism is clearly the same for the same class of $\mathrm{QD}$, whereas the difference in the slopes (parameter $B$, see text) represents the differences between the core-shell QDs (ZnS surface) and the uncapped QDs (CdSe surface).

same class of QD. The slope can be determined as $B=[(4.02 \pm$ $\left.0.20) \times 10^{4}\right] \mathrm{M} \mathrm{s}^{-1}$ when considering an abscissa value $k_{\mathrm{q} 0}=$ $\left[(1.2 \pm 0.3) \times 10^{6}\right] \mathrm{s}^{-1}$. The value $k_{\mathrm{q} 0}$ corresponds to a constant quenching rate that is not related to a wave function amplitude outside of the QD. In a similar consideration (neglecting $k_{\mathrm{q} 0}$ ), we obtain $B \approx 2.2 \times 10^{2} \mathrm{M} \mathrm{s}^{-1}$ for the uncapped CdSe QDs, which is about two orders of magnitude smaller than that for the core-shell CdSe/ZnS QDs (Figure 10B).

Without performing quantitative analysis, similar arguments hold for the high-molar-ratio regime. Here, analogously, the term $K_{c}^{\mathrm{d}}$ is a modified equilibrium constant. Interestingly, referring to the PL quenching of uncapped QDs in various absolute concentrations, its equal slope above $x_{c}$ in the Stern-Volmer plot (Figure $6 \mathrm{~B}$, inset) indicates that the underlying quenching processes are indeed governed by the elementary processes at the surface site, such as creation of surface sites through mobile TOPO ligands, ${ }^{[26]}$ which would generate new attachment sites for the dye. Figure $8 \mathrm{~A}$ shows that the maximum number of easily accessible sites per QD surface area is the same regardless of the size and type of shell of the QD. However, it indicates that the attachment probability can depend on the particular surface structure. We find the following three main reasons for the origin of the strong difference in $B$ for uncapped CdSe and core-shell CdSe/ZnS QDs:

1) The ZnS shell is not closed and is effectively only one monolayer thick or less. This enhances $\Psi^{2}$ at the attachment site, and thus, PL quenching with relation to the uncapped QD by more than one order of magnitude. ${ }^{[33]}$

2) Microscopic conditions are different for capped and uncapped QDs. One reason could be the formation of different (induced) surface states ${ }^{[59]}$ Note that, in the range of $x=1-$ 10, pyridine molecules do not quench PL in the case of CdSe/ZnS QDs, ${ }^{[33]}$ but are very effective quenchers in the case of uncapped CdSe QDs. This fact might indicate that even the wave function of the hole has be considered for uncapped QD because pyridine is a strong hole acceptor. ${ }^{[60]}$

3) The binding strength of pyridine with $\mathrm{Zn}^{2+}$ or $\mathrm{Cd}^{2+}$, and thus, the attachment probability for $\mathrm{H}_{2} \mathrm{P}$ might be different for capped and uncapped QDs.

Although there are some reasonable arguments for explanations of the clearly strong differences, a fully satisfying answer remains to be found. To summarise our model up to this point: each type of QD is assumed to initially have a number of free or easily (within $60 \mathrm{~s}$ ) accessible sites to allow the attachment of $\mathrm{H}_{2} \mathrm{P}$. As a consequence, at low $x$ ratios, attachment accompanied by quenching follows conventional Stern-Volmer behaviour. According to volume considerations, one $\mathrm{H}_{2} \mathrm{P}$ molecule in a perpendicular orientation ${ }^{[32,61]}$ replaces about three TOPO molecules, which are incorporated into a TOPO ligand shell. Above a critical value, $x_{c}$, which is significantly different for each type of QD, attachment sites probably have to be created through competition between detachment or rearrangement of TOPO and the attachment of $\mathrm{H}_{2} \mathrm{P}$.

\subsection{Ligand Dynamics}

As shown previously in several papers by our group ${ }^{[36]}$ and others, ${ }^{[8-12,45,47-50]}$ ligand dynamics play an essential role with respect to optical properties of QDs and have considerable impact on the QD quantum yield. Ji et al. determined, in the case of uncapped CdSe QDs $\left(d_{\mathrm{Cdse}}=3.5 \mathrm{~nm}\right)$, detachment rates, $k_{\text {det }}=0.01 \mathrm{~s}^{-1}$, and attachment rates, $k_{\mathrm{att}}=0.5 \mathrm{M}^{-1} \mathrm{~s}^{-1}$, for octylamine ligands $(\mathrm{OA})$ at concentrations of $[\mathrm{OA}]=0.01-0.1 \mathrm{M} \cdot{ }^{[8]}$ The corresponding equilibrium constant, $K^{\mathrm{L}}=k_{\text {att }} / k_{\text {det }}$, for the ligands is about $50 \mathrm{M}^{-1}$, compared with which, slower ${ }^{[49,50]}$ and also faster timescales have been reported. ${ }^{[9]}$ According to these results, ligands experience fast exchange between surface attachment and being in solution. In the case of OA on CdSe QDs, half of the ligand shell is exchanged within $70 \mathrm{~s}^{[8]}$

Upon preparation of extremely low concentration CdSe/ZnS capped with amine ligands, we observed a decrease in the PL 
with a decay time of about $1.2 \mathrm{s.}{ }^{[36]}$ This time is considerably longer than that observed by $\mathrm{Ji}$ et al., ${ }^{[8]}$ which might be due the addition of a $\mathrm{ZnS}$ shell that exhibits stronger ligand binding $^{[45]}$ and is also related to our findings when comparing the quenching efficiencies of $\mathrm{CdSe}$ and $\mathrm{CdSe} / \mathrm{ZnS}$ QDs. However, for TOPO ligands, this time may be considerably longer because the binding strength of TOPO is much stronger than that of amines. ${ }^{[45]} \mathrm{Ji}$ et al. ${ }^{[8]}$ also showed that the reduction of coverage of the QD surface by removing ligands is accompanied by a decrease in the PL intensity; this is also in line with observations by Munro et al. ${ }^{[12]}$ for PL quenching upon dilution. The decrease is either due to the creation of unsaturated bonds, causing a lower PL quantum yield. or due to an increased attachment of impurities and/or titrated quenchers. In case fast ligand exchange is also present in our experiments, a titration step is followed by two processes, namely, increased ligand desorption due to dilution and the replacement of ligands $(\mathrm{L})$ by quenchers $(\mathrm{M})$, in our case $\mathrm{H}_{2} \mathrm{P}$. In all of our experiments, the influence of dilution on the optical properties has been taken into account ${ }^{[32-37]}$ and can be neglected with respect to the influence of the dye and the expected ligand absorption.

A fast ligand exchange rate would be consistent with our titration experiments, since PL quenching is observed within $60 \mathrm{~s}$ of a single titration step. However, in case ligand exchange is much slower, the immediate PL response after titration points to the presence of a few "free" attachment sites, which correspond to the volume of about two to three TOPO ligands per $\mathrm{H}_{2} \mathrm{P}$ molecule. Depending on the binding strength and number of anchoring functional groups, the equilibrium constant, $K=k_{\text {att }} / k_{\text {det }}$, is different for ligands $\left(K^{\llcorner}\right)$and quencher molecules $\left(K^{\mathrm{M}}\right)$. This has been clearly proven in recent experiments, ${ }^{[32,34]}$ which revealed that the quenching efficiency at a given molar ratio depended drastically on the number and type of (ortho-, meta-, para-) pyridyl substituents of $\mathrm{H}_{2} \mathrm{P}$. For $(m \text {-Pyr })_{4} \mathrm{H}_{2} \mathrm{P}$ we have determined $K^{\mathrm{M}} \approx 10^{5}-10^{7} \mathrm{M}^{-1}$; a value that is about a factor of 4 smaller than that for $(\mathrm{Ph})_{3}(m-\mathrm{Pyr})_{1} \mathrm{H}_{2} \mathrm{P}^{[32,34]}$ $K^{\mathrm{M}}$ is thus at least 3 orders of magnitude larger than that of $K^{\mathrm{L}}=50 \mathrm{M}^{-1}$ observed for ligands in experiment by $\mathrm{Ji}$ et al. ${ }^{[8]}$ Due to the high value of $K^{\mathrm{M}}$, most $\mathrm{H}_{2} \mathrm{P}$ becomes attached to the QD surface over the course of time, at least at reasonably low concentrations. This finding can be discussed in light of spectral shifts of the $\mathrm{H}_{2} \mathrm{P}$ absorption (Soret band) upon titration of molar ratios of $x<1$ (see the Supporting Information), which accompany changes in the fluorescence decay times. ${ }^{[32,54]}$ Since $K^{\mathrm{M}} \gg K^{\mathrm{L}}$, we expect that, in the presence of a dye molecule at the QD surface, the ligand shell is almost completely replaced if the QD surface is assumed to be homogenous. In the case of a completely closed ligand shell, for any QD with a diameter of $d=3.5 \mathrm{~nm}$, the total average number of TOPO ligands per QD is close to $120 . .^{[37]}$ Following the same volume arguments ${ }^{[32,61]}$ as above, we expect that $\mathrm{H}_{2} \mathrm{P}$ would replace about 3 TOPO molecules, which would result in a maximum of about $40 \mathrm{H}_{2} \mathrm{P}$ molecules on the respective $\mathrm{QD}$ surface. We do not observe any influence on the $\mathrm{H}_{2} \mathrm{P}$ luminescence properties above about $x \approx 5$. From this fact, we conclude that no more than five $\mathrm{H}_{2} \mathrm{P}$ molecules can attach, at least not at such sites on the QD surface that give rise to spectral changes. However, as seen in Figure $7 \mathrm{~A}, \mathrm{PL}$ quenching still increases up to $x \approx 20$, although the spectral shifts have saturated (see the Supporting Information) and the fluorescent decay times remain constant, ${ }^{[32,54]}$ indicating a local replacement of TOPO by $\mathrm{H}_{2} \mathrm{P}$, as suggested by Voznyy. ${ }^{[26]}$ Again, from a comparison of ensemble and single-molecule experiments on PDI, we know that the number of PDI molecules attached to a QD is much less than that given by the molar ratio $x^{[37]}$ and we assume that a similar ratio also holds for $\mathrm{H}_{2} \mathrm{P}$. At very long waiting times $(>24 \mathrm{~h}){ }^{\left[{ }^{[3]}\right.}$ quenching still increases in the presence of $\mathrm{H}_{2} \mathrm{P}$, while also FRET increases (see Figure 5). From this we conclude that, at very high $\mathrm{H}_{2} \mathrm{P}$ concentrations and long waiting times, the TOPO ligand shell becomes almost completely replaced by $\mathrm{H}_{2} \mathrm{P}$ and the related value of $K^{\mathrm{M}}$ is much larger than that of $K^{L}$. These calculations from the titration experiments are in line with our suggested picture that the QD surface is inhomogeneous with respect to the attachment of quenching dye molecules.

To obtain more insight into this issue, Figure 5 can be inspected where the PL intensity of the QDs is shown as a function of time after sample preparation or after a titration step. Depending on the solvent conditions (TOPO concentration or, for example, water content), the PL intensity continuously decreases after sample preparation. This can be interpreted, as argued above, as a continuous loss of ligands. The decay time is shortest (150 s) for a large water content, but can be increased by adding extra TOPO (600 s). On the other hand, starting with dried toluene, the decay time becomes longer ( $>$ $30 \mathrm{~min}$ ) and is not influenced by adding extra TOPO. This effect can be understood as a combination of a permanent loss of ligands accompanied by a decrease of PL due to impurities present in the solvent (e.g., water). A higher ligand concentration results in higher surface coverage. On the other hand, when removing inherent impurities, the absolute ligand concentration becomes much less important.

What happens, however, when adding $\mathrm{H}_{2} \mathrm{P}$ in a one-step titration? This is also shown in Figure 5 for concentrations of $1 \times$ $10^{-7}, 2 \times 10^{-7}$ and $4 \times 10^{-7} \mathrm{M}$ of uncapped CdSe QDs. The double logarithmic plot shows that, at least for one order in time, decay is no longer non-exponential, but nearly follows a power law. This observation implies that $\mathrm{PL}$ quenching becomes more complex and is even partly reduced for $\mathrm{H}_{2} \mathrm{P}$-containing samples. It can be ascribed to the high complexation constant for $\mathrm{H}_{2} \mathrm{P}$, which may block sites that are subject to an otherwise unknown strong quenching mechanism. Further information is obtained when inspecting the FRET efficiency as a function of time. On the timescale of our experiment, after an initial increase, the FRET efficiency becomes stronger for some time and reaches a final value that decays again in the case of the lowest QD concentration of $1 \times 10^{-7} \mathrm{M}$. This behaviour can be understood by assuming that not all $\mathrm{H}_{2} \mathrm{P}$ molecules become attached immediately to the $\mathrm{QD}$, but become attached on timescales of about $600 \mathrm{~s}$; a time that is close to the one observed in the case of the slowly decaying PL intensity. Figure 5 reveals that the PL intensity decrease following a titration step is a function of $x$ itself. When $x$ increases, the appar- 
ent decay time becomes shorter and the quenching increases as well. This observation implies that $\mathrm{H}_{2} \mathrm{P}$ molecules originally remaining in solution become attached to the $\mathrm{QD}$ surface and is accompanied by increasing FRET. When waiting for longer than $24 \mathrm{~h}, \mathrm{PL}$ decreases further and is accompanied again by an increase in FRET. Also, from these observations, we conclude that a majority of $\mathrm{H}_{2} \mathrm{P}$ becomes attached to the $\mathrm{QD}$ surface at longer times and effectively replaces a large fraction of the original TOPO ligands. However, the time behaviour is clearly characterised by a broad distribution of detachment and attachment processes, which may result from complex ligand exchange or ligand reorganisation processes on the QD surface. Moreover, PL quenching may also be caused by unknown quenchers and permanent PL quenching, for example, due to photo-oxidation. Since these processes may depend on several partly hidden conditions, such as QD surface structure and absolute TOPO concentration, they vary from sample to sample and QD to QD. However, though absolute values differ from each other considerably, we observed the same qualitative behaviour in all cases.

The above-described effects are much less pronounced with $n$-octane as a solvent (see the Supporting Information). This is easily understood because the solubility of TOPO is largely reduced in $n$-octane, while that of $\mathrm{H}_{2} \mathrm{P}$ increases compared with in toluene. This shifts the equilibrium constants $K^{\mathrm{M}}$ and $K^{\mathrm{L}}$ towards each other. In that case, the ligand shell remains intact and prevents $\mathrm{H}_{2} \mathrm{P}$ from becoming attached effectively. The opposite result is observed when increasing the polarity of the solvent: when adding acetone to the solution in toluene, PL quenching is observed for both pure QDs (down to $20 \%$ ) and $\mathrm{QD}-\mathrm{H}_{2} \mathrm{P}$ assemblies (down to $40 \%$ ) at $x=5$. $^{\text {[2] }}$

\section{Conclusions}

We have shown that PL quenching of CdSe and CdSe/ZnS QDs by functionalised porphyrin molecules as quenchers can be explained by the presence of a limited number of empty attachment sites on the QD surface, followed by ligand exchange on much slower timescales: after saturating the empty attachment sites, the quencher molecules may be attached to the QD surface only after reorganisation of the ligand shell. Related experiments with TOPO ligands and CdSe/ZnS QDs have, to the best of our knowledge, not yet been reported. In particular, the non-FRET part of PL quenching induced by the quencher molecule relates to ligand replacement, which is different for the different chemical properties of the attachment sites. We are convinced that the immediate $\mathrm{PL}$ quenching, after a one-step titration, reported herein follows the same general rules as those reported by Ji et al. with respect to amine ligand dynamics. ${ }^{[8]}$ Instead of investigating ligand dynamics directly, for example, by NMR spectroscopy, we suggest making use of $\mathrm{PL}$ quenching in combination with appropriately functionalised dye molecules. In contrast to NMR spectroscopy, this approach allows the investigation of ligand dynamics at extremely low concentrations of concurrent ligand-type dyes.

Since sample preparation is accompanied by diluting the original QD solution in toluene followed by a new equilibrium of TOPO coverage, an overall reduction in the QD surface coverage by TOPO ligands is implied. This fact results in a decrease in PL quantum yield due to the formation of unsaturated surface states. ${ }^{[8,27]}$ In addition, it allows the attachment of quenchers, either those already inherently present or those added during a titration procedure. The timescale of such processes is typically between 200 and $2000 \mathrm{~s}$ in the present experiments and depends critically on the experimental conditions, such as the type of QD, TOPO, unknown impurities and/ or dye concentration. Of crucial importance is the kind of solvent. Less polar solvents, such as n-octane, clearly prevent depletion of TOPO from the QD surface more effectively than toluene. This is understood according to recent calculations, which show that ligand binding occurs through electrostatic forces $;{ }^{[45]}$ these are reduced in polar solvents. However, in addition to processes on a timescale below $2000 \mathrm{~s}$, a further reorganisation of the surfactant shell (accompanied by increased quenching) occurs on much longer timescales. ${ }^{[34]}$ We do not yet have an explanation for this finding, but it might be related to the formation of new TOPO structures, such as surfactant islands on the QD surface or TOPO micelles in solution. In principle, according to $\mathrm{Yu}$ et al. ${ }^{\left[{ }^{[62]}\right]}$ the configuration of even parts of the ligands on the surface of semiconductor nanocrystals plays a critical role for their stability. Another plausible explanation can be related to oxidative degradation of the QD surface on these long timescales, as reported by van Sark et al. ${ }^{[63]}$ Such effects were reported by $\mathrm{us}^{[36,37,64]}$ Peng and co-workers ${ }^{[8]}$ and Meijerink and co-workers ${ }^{[9]}$ in the case of amine ligands, in particular, for properly designed PDI molecules. ${ }^{[64]}$ However, we did not observe significant changes in the QD absorption and/ or PL spectra in the case of TOPO ligands. We would also like to emphasise that, at these long timescales, there is increasing attachment of $(m-\mathrm{Pyr})_{4}-\mathrm{H}_{2} \mathrm{P}$, as identified by increasing FRET. All of the above-described effects are much more evident for uncapped QDs.

Our present findings of inhomogeneous surface dynamics are also important for non-FRET quenching processes reported recently. ${ }^{[32,33,36]}$ We have argued that non-FRET quenching might be related to depletion of ligands by the respective dye molecules-PDI in those cases. However, the related number of ligands removed that were necessary to explain the observed non-FRET efficiency was much too large with respect to the volume of the dye molecule. Herein, we suggest that such an non-specified quenching mechanism ${ }^{[24]}$ could be related to the inhomogeneity of the QD surface with respect to ligand attachment. In case there are various attachments sites with varying binding strengths, it is reasonable to assume that different attachment sites are correspondingly related to the formation of trap states, which quench the QD PL effectively. The presence of various, partial ligand-related trap states has been suggested by numerical calculations. ${ }^{[26,27]}$ Summarising all of these arguments, non-FRET quenching corresponds to the removal of ligands at specific surface sites and the creation of trap states that lead to PL quenching. Hence, tuning the PL properties does not require full exchange of the ligand shell; it already works upon attachment of a few dye molecules. 


\section{Experimental Section}

\section{CdSe Quantum Dots}

The colloidal TOPO-capped CdSe and CdSe/ZnS QDs (uncapped CdSe and CdSe/ZnS core-shell) were obtained from Evident Technologies (Troy, NY, USA). The molar absorption coefficients and core diameters of the QDs were calculated from the first exciton absorption peak, according to Yu et al. ${ }^{[65]}$ The absorbance of the QD starting solutions was adjusted to less than $0.1 \mathrm{OD}$ at excitation and emission wavelengths to avoid non-linear absorption and reabsorption effects. The concentrations varied over the range 1$10 \times 10^{-7} \mathrm{M}$. The PL stability of the QD solutions was checked while obtaining a constant quantum yield for at least $3 \mathrm{~h}$ after preparation. The properties of the used QDs are found in the Supporting Information (Table A.I).

\section{Pyridyl-Functionalised Porphyrin}

$(m-\mathrm{Pyr})_{4}-\mathrm{H}_{2} \mathrm{P}$ has been used to form assemblies both with $\mathrm{CdSe}$ QDs and CdSe core-shell QDs (covered with 2 monolayers (ML) $\mathrm{ZnS})$. ( $m-\mathrm{Pyr})_{4}-\mathrm{H}_{2} \mathrm{P}$ was synthesised according to established protocols. ${ }^{[66,67]}$ The porphyrin stock solution was prepared in toluene under ultrasonic treatment at $40^{\circ} \mathrm{C}$ at concentrations in the range $(3-30) \times 10^{-5} \mathrm{M}$ such as those reported previously by us. ${ }^{[32-36,61,68-72]}$

\section{Titration and Formation of Nanoassemblies}

Titration experiments were carried out under ambient conditions by adding small aliquots $(5-10 \mu \mathrm{L})$ of $(m-\mathrm{Pyr})_{4}-\mathrm{H}_{2} \mathrm{P}$ to the $\mathrm{QD}$ master solution $(2.6 \mathrm{~mL})$. For quantitative titration experiments, spectroscopic-grade toluene (Fluka SeccoSolv dried with a molecular sieve) was used. Optical cuvettes (Hellma QS-111, path length $1 \mathrm{~cm}$ ) and other glassware were flushed with acetone and ethanol, chemically cleaned with aqueous $\mathrm{H}_{2} \mathrm{SO}_{4} / \mathrm{H}_{2} \mathrm{O}_{2}$, flushed with deionised water, dried in a nitrogen flow and purged with toluene. In the titration experiments, the absorption and emission spectra of $\mathrm{QD}$ and solutions of $(m-\mathrm{Pyr})_{4}-\mathrm{H}_{2} \mathrm{P}$ were recorded with a Shimadzu 3001 UV/Vis spectrometer and a Shimadzu RF-5001PC spectrofluorophotometer, respectively. By titration, molar ratios in the range $\left.x=\left[(m-\mathrm{Pyr})_{4}-\mathrm{H}_{2} \mathrm{P}\right] /[\mathrm{QD}]\right)=0.1-20$ were obtained. For the PL spectra, to keep direct excitation of $\left(m-\mathrm{Pyr}_{4}\right)_{4}-\mathrm{H}_{2} \mathrm{P}$ as low as possible, the QDs were excited at $465 \mathrm{~nm}$, that is, in the porphyrin absorption minimum between the Soret- and Q-band absorption. For quantitative comparison and reproducibility, it was necessary to perform experiments under exactly the same procedures of sample preparation. The residual QD emission intensity was numerically separated. The PL quantum efficiencies were determined relative to a quininsulfate dihydrate standard. Optical properties of the QD in toluene were determined for a series of samples under ambient conditions (see ref. [54] for a comprehensive description).

\section{Acknowledgements}

We acknowledge financial support by the Volkswagen foundation (Priority program "Physics, Chemistry and Biology with Single Molecules", grant I/79435 to T.B. and M.A.-M.), the German Science Foundation (DFG, Graduate College 829 to T.B.), INTAS (grant R.03-05-4540 to E.I.Z.), BRFFI (grant $\Phi 10 C O-005:$ E.I.Z.), and the German Academic Exchange Service (DAAD, grant 325 to E.I.Z. and RISE program 2005 to K.S.).
Keywords: FRET - ligand effects - photoluminescence quenching $\cdot$ quantum dots $\cdot$ self-assembly $\cdot$ semiconductors

[1] S. V. Gaponenko, Optical Properties of Semiconductor Nanocrystals, Cambridge University Press, Cambridge, 1998.

[2] Single Semiconductor Quantum Dots (Ed.: P. Michler), Springer, Heidelberg, 2009.

[3] Semiconductor Nanocrystal Quantum Dots: Synthesis Assembly, Spectroscopy and Applications (Ed.: A. L. Rogach), Springer, Heidelberg, 2008.

[4] A. L. Efros, M. Rosen, Annu. Rev. Mater. Sci. 2000, 30, 475

[5] P. Reiss, M. Protiere, L. Li, Small 2009, 5, 154.

[6] A. J. Morris-Cohen, M. D. Donakowski, K. E. Knowles, E. A. Weiss, J. Phys. Chem. C 2010, 114, 897.

[7] C. M. Evans, M. E. Evans, T. D. Krauss, J. Am. Chem. Soc. 2010, 132, 10973.

[8] X. Ji, D. Copenhaver, C. Sichmeller, X. Peng, J. Am. Chem. Soc. 2008, 130, 5726.

[9] R. Koole, P. Schapotschnikow, C. de Mello Donega, T. J. H. Vlugt, A. Meijerink, ACS Nano 2008, 2, 1703.

[10] G. Kalyuzhny, R. W. Murray, J. Phys. Chem. B 2005, 109, 7012.

[11] A. M. Munro, D. S. Ginger, Nano Lett. 2008, 8, 2585.

[12] A. M. Munro, I. Jen-La Plante, M. S. Ng, D. S. Ginger, J. Phys. Chem. C 2007, 111, 6220.

[13] C. Querner, P. Reiss, J. Bleuse, A. Pron, J. Am. Chem. Soc. 2004, 126, 11574.

[14] S. Dayal, Y. Lou, A. C. S. Samia, J. C. Berlin, M. E. Kenney, C. Burda, J. Am. Chem. Soc. 2006, 128, 13974.

[15] T. C. Lim, V. J. Bailey, Y.-P. Ho, T.-H. Wang, Nanotechnology 2008, 19, 075701.

[16] T. Ren, P. K. Mandal, W. Erker, Z. Liu, Y. Avlasevich, L. Puhl, K. Müllen, T. Basché, J. Am. Chem. Soc. 2008, 130, 17242.

[17] C. Bullen, P. Mulvaney, Langmuir 2006, 22, 3007.

[18] K. E. Knowles, D. B. Tice, E. A. McArthur, G. C. Solomon, E. A. Weiss, J. Am. Chem. Soc. 2010, 132, 1041.

[19] R. Jin, Angew. Chem. 2008, 120, 6852-6855; Angew. Chem. Int. Ed. 2008 47,6750 .

[20] I. Gur, N. A. Fromer, C.-P. Chen, A. G. Kanaras, A. P. Alivisatos, Nano Lett. 2007, 7, 409.

[21] H. S. Mansur, A. A. P. Mansur, Mater. Chem. Phys. 2011, 125, 709.

[22] B. von Holt, S. Kudera, A. Weiss, T. E. Schrader, L. Manna, W. J. Parak, M. Braun, J. Mater. Chem. 2008, 18, 2728.

[23] O. Schmelz, A. Mews, T. Basché, A. Herrmann, K. Müllen, Langmuir 2001, 17, 2861.

[24] D. M. Willard, L. L. Carillo, J. Jung, A. van Orden, Nano Lett. 2001, 1, 469.

[25] F. J. Schmitt, J. Opt. 2010, 12, 084008.

[26] O. Voznyy, J. Phys. Chem. C 2011, 115, 15927.

[27] J. Frenzel, J.-O. Joswig, G. Seifert, J. Phys. Chem. C 2007, 111, 10761.

[28] M. Califano, A. Franceschetti, A. Zunger, Nano Lett. 2005, 5, 2360.

[29] A. Issac, C. von Borczyskowski, F. Cichos, Phys. Rev. B 2005, 71, R161302.

[30] F. C. Krebs, V. Senkovskyy, A. Kiriy, IEEE J. Sel. Top. Quantum Electron. 2010, 16, 1821

[31] N. Marjanovic, J. Hammerschmidt, J. Perelaer, S. Farnsworth, I. Rawson, M. Kus, E. Yenel, S. Tilki, U. S. Schubert, R. R. Baumann, J. Mater. Chem. 2011, 21, 13634.

[32] E. I. Zenkevich, A. M. Shulga, F. Cichos, E. Petrov, T. Blaudeck, C. von Borczyskowski, J. Phys. Chem. B 2005, 109, 8679.

[33] T. Blaudeck, E. I. Zenkevich, F. Cichos, C. von Borczyskowski, J. Phys. Chem. C 2008, 112, 20251.

[34] E. I. Zenkevich, T. Blaudeck, M. M. S. Abdel-Mottaleb, F. Cichos, A. M. Shulga, C. von Borczyskowski, Int. J. Photoenergy 2006, 90242.

[35] E. I. Zenkevich, T. Blaudeck, A. M. Shulga, F. Cichos, C. von Borczyskowski, J. Lumin. 2007, 122, 784.

[36] D. Kowerko, J. Schuster, N. Amecke, M. Abdel-Mottaleb, R. Dobrawa, F. Würthner, C. von Borczyskowski, Phys. Chem. Chem. Phys. 2010, 12, 4112.

[37] D. Kowerko, S. Krause, N. Amecke, M. Abdel-Mottaleb, J. Schuster, C. von Borczyskowski, Int. J. Mol. Sci. 2009, 10, 5239.

[38] J. R. Lakowicz, Principles of Fluorescence Spectroscopy, 2nd ed., Kluwer, New York, 1999. 
[39] T. Förster, Fluoreszenz Organischer Verbindungen, Vandenhoeck \& Rupprecht, Göttingen, 1951.

[40] A. Javier, D. Magana, T. Jennings, G. F. Strouse, Appl. Phys. Lett. 2003, 83, 1423.

[41] E. I. Zenkevich, C. von Borczyskowski, Macroheterocycles 2009, 2, 206.

[42] A. V. Chernook, A. M. Shulga, E. I. Zenkevich, U. Rempel, C. von Borczyskowski, J. Phys. Chem. 1996, 100, 1918.

[43] C. von Borczyskowski, U. Rempel, E. I. Zenkevich, A. M. Shulga, A. V. Chernook, J. Mol. Struct. 1995, 348, 441.

[44] C. Krasselt, J. Schuster, C. von Borczyskowski, Phys. Chem. Chem. Phys. 2011, 13, 17084.

[45] P. Schapotschnikow, B. Hommersom, T. J. H. Vlugt, J. Phys. Chem. C 2009, 113, 12690.

[46] G. C. Schatz, A. Kuppermann, J. Chem. Phys. 1976, 65, 4668.

[47] A. Mews, Z. Phys. Chem. 2007, 221, 295.

[48] S. Trotzky, J. Kolny-Olesiak, S. M. Falke, T. Hoyer, C. Lienau, W. Tuszynski, J. Parisi, J. Phys. D 2008, 41, 102004.

[49] N. Pradhan, D. Reifsnyder, R. Xie, J. Aldana, X. Peng, J. Am. Chem. Soc. 2007, 129, 9500

[50] Z. Ning, M. Molnár, Y. Chen, P. Friberg, L. Gan, H. Ågren, Y. Fu, Phys. Chem. Chem. Phys. 2011, 13, 5848.

[51] E. I. Zenkevich, T. Blaudeck, M. Heidernätsch, F. Cichos, C. von Borczyskowski, Theor. Exp. Chem. 2009, 45, 23.

[52] E. I. Zenkevich, A. M. Shulga, C. von Borczyskowski, U. Rempel, J. Appl. Spectrosc. 1995, 62, 93.

[53] E. I. Zenkevich, C. von Borczyskowski, Photoinduced relaxation processes in self-assembled nanostructures: multiporphyrin complexes and composites CdSe/ZnS quantum dot-porphyrin in Multiporphyrin Arrays: Fundamentals and Applications (Ed.: D. Kim), Pan Stanford Publishing Pte. Ltd., Singapore, 2011, pp. $181-252$.

[54] T. Blaudeck, Ph.D. Thesis, TU Chemnitz, 2007.

[55] H. C. Wolf, H. Port, J. Lumin. 1976, 12-13, 33

[56] J. Y. Rempel, B. L. Trout, M. G. Bawendi, K. F. Jensen, J. Phys. Chem. B 2005, 109, 19320
[57] J. Y. Rempel, B. L. Trout, M. G. Bawendi, J. Phys. Chem. B 2006, 110 18007.

[58] R. Schuster, M. Barth, A. Gruber, F. Cichos, Chem. Phys. Lett. 2005, 413, 280.

[59] E. P. Petrov, F. Cichos, C. von Borczyskowski, J. Lumin. 2006, 119-120, 412.

[60] V. I. Klimov, A. A. Mikhailovsky, D. W. McBranch, C. A. Leatherdale, M. G. Bawendi, Phys. Rev. B 2000, 61, R13349.

[61] E. I. Zenkevich, A. M. Shulga, C. von Borczyskowski, J. Porphyrins Phthalocyanines 2003, 7, 731.

[62] W. W. Yu, Y. A. Yang, X. Peng, Chem. Mater. 2003, 15, 4300.

[63] W. G. J. H. M. van Sark, P. L. T. M. Frederix, D. J. V. den Heuvel, H. C. Gerritsen, A. A. Bol, J. N. J. van Lingen, C. de Mello Donega, A. Meijerink, J. Phys. Chem. B 2001, 105, 8281.

[64] D. Kowerko, Ph.D. Thesis, TU Chemnitz, 2010.

[65] W. W. Yu, L. H. Qu, Z. Guo, X. G. Peng, Chem. Mater. 2003, 15, 2854.

[66] F. R. Longo, M. G. Finarelli, J. B. Kim, J. Heterocycl. Chem. 1969, 6, 927.

[67] R. G. Little, J. A. Anton, P. A. Loach, J. A. Ibers, J. Heterocycl. Chem. 1975, $12,343$.

[68] E. I. Zenkevich, A. M. Shulga, S. Bachilo, U. Rempel, A. Willert, C. von Borczyskowski, Chem. Phys. 2002, 275, 185.

[69] E. I. Zenkevich, C. von Borczyskowski, A. M. Shulga, J. Porphyrins Phthalocyanines 2003, 7, 731 .

[70] E. I. Zenkevich, E. I. Sagun, A. A. Yarovoi, A. M. Shulga, V. N. Knyukshto, A. P. Stupak, C. von Borczyskowski, Opt. Spectrosc. 2007, 103, 958.

[71] E. I. Zenkevich, C. von Borczyskowski, A. M. Shulga, S. M. Bachilo, U. Rempel, A. Willert, Chem. Phys. 2002, 275, 185.

[72] E. I. Zenkevich, E. I. Sagun, V. N. Knyukshto, A. S. Stasheuski, V. A. Galievsky, A. P. Stupak, T. Blaudeck, C. von Borczyskowski, J. Phys. Chem. 2011, 115, 21535

Received: September 16, 2011

Published online on December 23, 2011 